\title{
Radiation from Wide-Angle Conical Antennas Fed by a Coaxial Line*
}

\author{
CHARLES H. PAPAS $\dagger$, ASSOCIATE, IRE, AND RONOLD KING $\dagger$, SENIOR MEMBER, IRE
}

Summary-An approximate expression for the radiation from spherically capped conical antennas is derived by the Fourier-Lamé eigen-function method. Radiation patterns have been calculated for antennas with flare angle of $\pi / 6$ and various lengths.

\section{INTRODUCTION}

I N A PREVIOUS PAPER ${ }^{1}$ the input impedances of spherically capped conical antennas fed by a coaxial line were calculated for large flare angles. By an extension of this earlier work an expression for the electromagnetic field of an antenna of arbitrary length $a$ and flare angle $\theta_{0}$ (see Fig. 1) is obtained. It is sup-

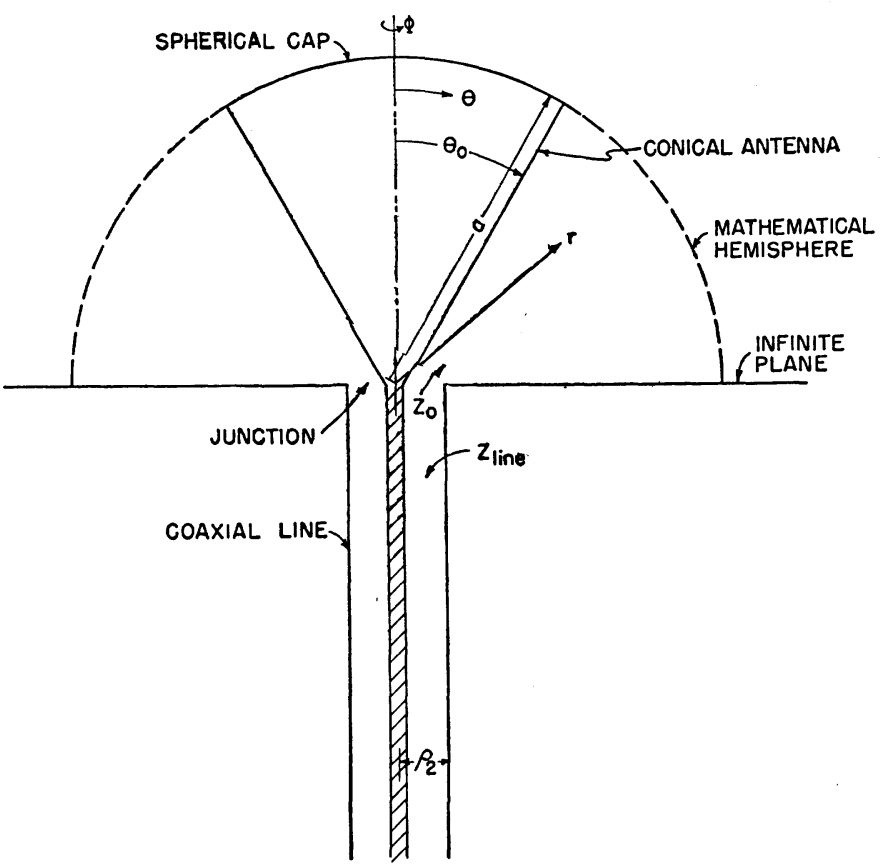

Fig. 1-Spherically capped conical antenna fed by coaxial feed line. Characteristic impedance of line equals characteristic impedance of antenna and flare angle is large, i.e., $\theta_{0} \geqq \pi / 6$.

posed that the characteristic impedance of the coaxial line

$$
Z_{\text {line }}=60 \ln \frac{\rho_{2}}{\rho_{1}},
$$

where $\rho_{2}$ and $\rho_{1}$ are respectively the radii of its outer and inner conductors, is equal to the characteristic impedance of the antenna

* Decimal classification: R120 $\times$ R326.612. Original manuscript received by the Institute, January $16,1950$.

The research reported in this paper was made possible through support extended Cruft Laboratory, Harvard University, jointly by the Navy Department (Office of Naval Research), the Signal Corps of the U. S. Army, and the U. S. Air Force, under ONR Contract N5ori-76, T. O. 1.

$\dagger$ Cruft Laboratory, Harvard University, Cambridge, Mass.

i C. H. Papas and R. King, "Input impedance of wide-angle conical antennas fed by a coaxial line," Proc. I.R.E., vol. 37, pp. 12691271; November, 1949.

$$
Z_{0}=60 \ln \cot \frac{\theta_{0}}{2}
$$

From symmetry considerations it is known that the only nonvanishing components of the electromagnetic field in the antenna region $\rho_{2} \leqq r \leqq a, \theta_{0} \leqq \theta \leqq \pi / 2$, and the radiation region $r \geqq \mathrm{a}, 0 \leqq \theta \leqq \pi / 2$ are $H_{\phi}, E_{\theta}, E_{r}$. Assuming that only the TEM mode exists in the antenna region, and expanding the field in the radiation region in an infinite series of eigen-functions, the radiation field is determined by forcing the annihilation of $E_{\theta}$ over the spherical cap and the continuity of $E_{\theta}$ over the mathematical surface $r=a, \theta_{0} \leqq \theta \leqq \pi / 2$.

\section{Formulation of the Solution}

In the antenna region for large flare angles, i.e., $\theta_{0} \geqq \pi / 6$, only the $T E M$ mode need be considered. The $\theta$ component of the electric field for this TEM mode is given by the following expression: ${ }^{2}$

$$
E_{\theta}(r, \theta)=\sqrt{\frac{\mu}{\epsilon}} \frac{\alpha e^{-i k r}-\beta e^{i k r}}{r \sin \theta}
$$

where $\alpha$ and $\beta$ are constants, $k=2 \pi \div$ free-space wavelength.

In the radiation region, it is easily shown that ${ }^{2}$

$$
\begin{aligned}
E_{\theta}(r, \theta)=i \sqrt{\frac{\bar{\mu}}{\epsilon} \sum_{n=1}^{\infty} A_{n}}\left[h_{n-1}{ }^{(2)}(k r)\right. \\
\left.-\frac{n}{k r} h_{n}{ }^{(2)}(k r)\right] P_{n}{ }^{1}(\cos \theta),
\end{aligned}
$$

where $A_{n}$ 's are constants, $h_{n}{ }^{(2)}(x)$ are the spherical Hankel functions of the second kind, $P_{n}{ }^{1}(\cos \theta)=-d / d \theta P_{n}$ $(\cos \theta)$ are the associated Legendre polynomials, and the summation is over odd integers.

Since $E_{\theta}(a, \theta)$ must vanish over the spherical cap, from (2) it follows that

$$
0=\sum_{n=1}^{\infty} A_{n}\left(h_{n-1}{ }^{(2)}(k a)-\frac{n}{k a} h_{n}^{(2)}(k a)\right) P_{n}{ }^{1}(\cos \theta)
$$

for $0 \leqq \theta \leqq \theta_{0}$. To match $E_{\theta}$ across the mouth of the antenna, i.e., across the mathematical surface, $r=a$, $\theta_{0} \leqq \theta \leqq \pi / 2$, it is necessary to satisfy the relation

$$
\begin{aligned}
& \frac{\alpha e^{-i k a}-\beta e^{i k a}}{a} \sin \theta \\
& \quad=i \sum_{n=1}^{\infty} A_{n}\left(h_{n-1}{ }^{(2)}(k a)-\frac{n}{k a} h_{n}{ }^{(2)}(k a)\right) P_{n}{ }^{1}(\cos \theta)
\end{aligned}
$$

${ }^{2}$ C. H. Papas and R. King, "Input Impedance of Wide-Angle Conical Antennas," Cruft Laboratory Technical Report No. 52, December 1, 1948. 
obtained by equating (1) and (2) for $r=a$. Multiplying both sides of (3) and (4) by $P_{n}{ }^{1}(\cos \theta) \sin \theta$ and integrating with respect to $\theta$ over their respective intervals of validity, it follows from the orthogonality property of the associated Legendre polynomials that

$$
\begin{aligned}
& \left(\frac{\alpha e^{-i k a}-\beta e^{i k a}}{a}\right) P_{n}\left(\cos \theta_{0}\right) \\
& \quad=i A_{n}\left[h_{n-1}{ }^{(2)}(k a)-\frac{n}{k a} h_{n}{ }^{(2)}(k a)\right] \frac{n(n+1)}{2 n+1} .
\end{aligned}
$$

Solving (5) for the $A_{n}$ 's and then substituting into (2), the $\theta$ component of the electric field in the radiation region is obtained

\section{Radiation Patterns}

For given values of the flare angle $\theta_{0}$, the antenna length $a$, and the operating angular frequency $\omega(=k c$ where $c$ is the velocity of light in vacuum), the radiation pattern of the antenna can be computed from (8). When $k a \ll 1$, only the first terms in the series need be considered and consequently

$$
M(\theta)=\sin \theta
$$

which is to be expected for the radiation pattern of an infinitesimal vertical dipole on a perfectly conducting horizontal plane. As the parameter $k a$ increases, the

$$
E_{\theta}(r, \theta)=K \sum_{n=1}^{\infty} P_{n}\left(\cos \theta_{o}\right) P_{n}{ }^{1}(\cos \theta) \frac{2 n+1}{n^{2}+n} \frac{h_{n-1}{ }^{(2)}(k r)-(n / k r) h_{n}{ }^{(2)}(k r)}{h_{n-1}{ }^{(2)}(k a)-(n / k a) h_{n}{ }^{(2)}(k a)}
$$

where

$$
K=\sqrt{\frac{\bar{\mu}}{\epsilon}}\left(\frac{\alpha e^{-i k a}-\beta e^{i k a}}{a}\right) .
$$

At distances from the antenna large compared to a wavelength, i.e., $k r \rightarrow \infty$, the expression $\left[h_{n-1}{ }^{(2)}(k r)\right.$ $\left.-(n / k r) h_{n}{ }^{(2)}(k r)\right]$ approaches $(i)^{n} e^{-i k r} / k r$. Hence for $k r \rightarrow \infty$ departure from (9) becomes more pronounced as is seen from the polar plots of Fig. 2 for the case $\theta_{0}=\pi / 6$.

\section{ACKNOWLEDGMENT}

The authors gratefully acknowledge the aid of Miss J. Klimas who performed all the computations.

$$
E_{\theta}(r, \theta)=K \frac{e^{-i k r}}{r} \sum_{n=1}^{\infty} P_{n}\left(\cos \theta_{o}\right) P_{n}{ }^{1}(\cos \theta) \frac{2 n+1}{n^{2}+n} \frac{i^{n}}{h_{n-1}{ }^{(2)}(k a)-(n / k a) h_{n}{ }^{(2)}(k a)} .
$$

The expression (7) yields the $\theta$ component of the electric field along the circle $r, \theta$ when $k r \gg 1$. Dividing (7) by $\left(E_{\theta}(r, \pi / 2)\right.$ yields the normalized radiation distribution function

$$
R(\theta)=\frac{E_{\theta}(r, \theta)}{E_{\theta}\left(r, \frac{\pi}{2}\right)}=\frac{\sum_{n=1}^{\infty} P_{n}\left(\cos \theta_{0}\right) P_{n}{ }^{1}(\cos \theta) \frac{2 n+1}{n^{2}+n} i^{n}\left[h_{n-1}{ }^{(2)}(k a)-(n / k a) h_{n}^{(2)}(k a)\right]^{-1}}{\sum_{n=1}^{\infty} P_{n}\left(\cos \theta_{0}\right) P_{n}{ }^{1}(0) \frac{2 n+1}{n^{2}+n} i^{n}\left[h_{n-1}{ }^{(2)}(k a)-(n / k a) h_{n}{ }^{(2)}(k a)\right]^{-1}} .
$$

$R(\theta)$ is a complex function of $\theta$ whose modulus $M(\theta)$ gives the distribution of the absolute value of the normalized electric field over a hemisphere of very large radius. Explicitly,

$$
M(\theta)=\frac{\left|\sum_{n=1}^{\infty} P_{n}\left(\cos \theta_{0}\right) P_{n}{ }^{1}(\cos \theta) \frac{2 n+1}{n^{2}+n} i^{n}\left[h_{n-1}{ }^{(2)}(k a)-(n / k a) h_{n}{ }^{(2)}(k a)\right]^{-1}\right|}{\left|\sum_{n=1}^{\infty} P_{n}\left(\cos \theta_{o}\right) P_{n}{ }^{1}(0) \frac{2 n+1}{n^{2}+n} i^{n}\left[h_{n-1}{ }^{(2)}(k a)-(n / k a) h_{n}{ }^{(2)}(k a)\right]^{-1}\right|} .
$$




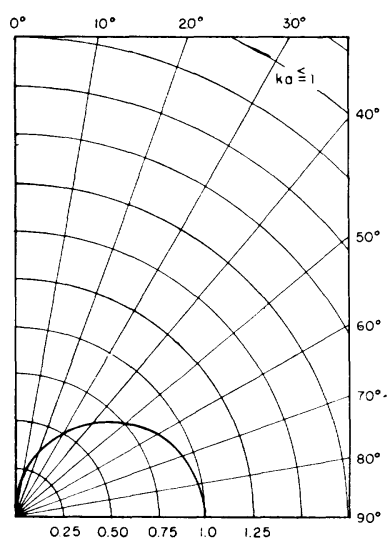

(a)

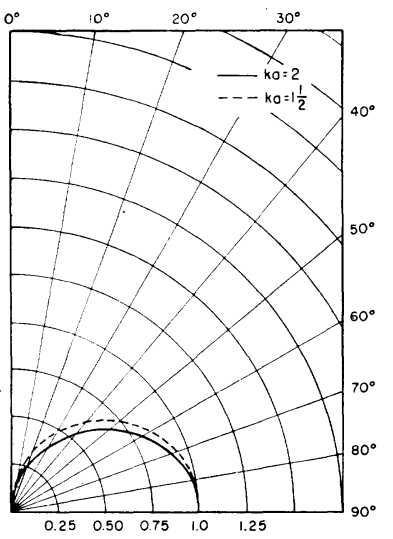

(b)

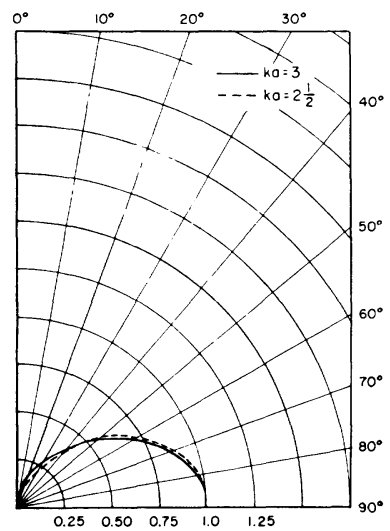

(c)

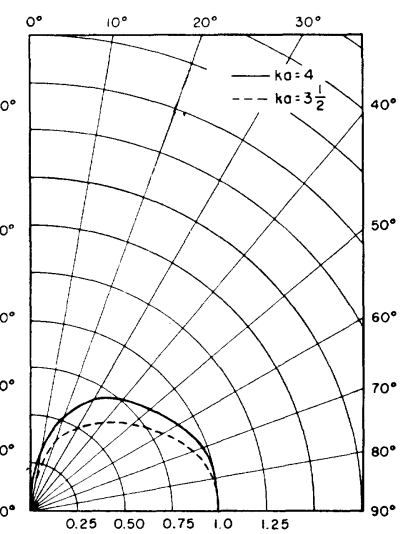

(d)

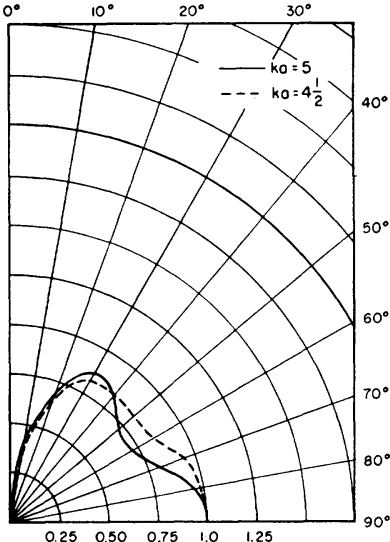

(e)

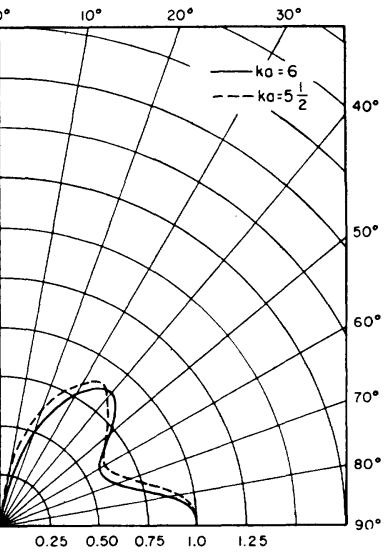

(f)

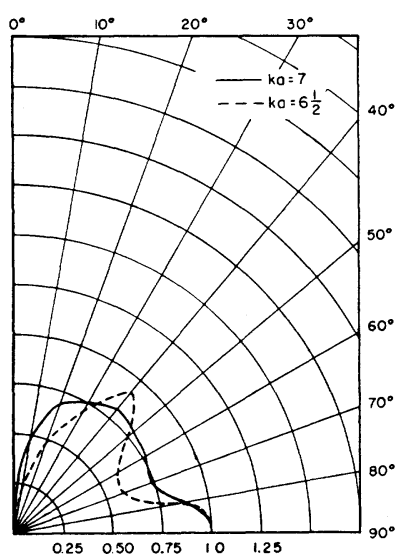

(g)

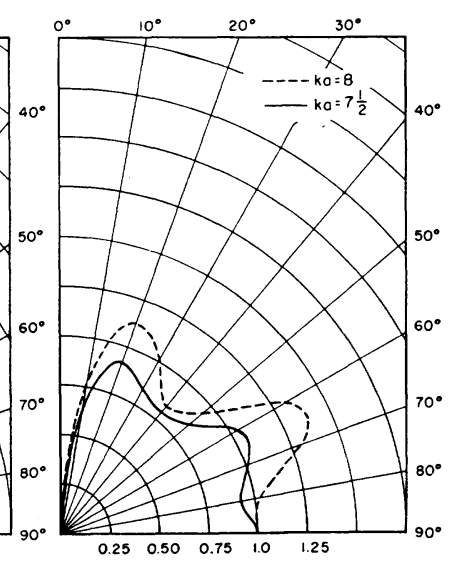

(h)

Fig. 2-Plots of the absolute values of the far-zone electric field as a function of the zenithal angle $\theta$ for various values of $k a$ and flare angle $\theta_{0} \cdot k a=2 \pi x$ length of antenna in free-space wavelengths.

\title{
Angular Jitter in Conventional Conical-Scanning, Automatic-Tracking Radar Systems ${ }^{*}$
}

\author{
CHARLES E. BROCKNER $†$, SENIOR MEMBER, IRE
}

\begin{abstract}
Summary-Four sources of angular jitter in a conventional conical-scanning, automatic-tracking radar are discussed and each is expressed in terms of range and the several system parameters. The total-angular-jitter-versus-range curve is shown to have a characteristic shape exhibiting a certain range interval of optimum tracking. The importance of beamwidth in determining the magnitude of angular jitter is also stressed.
\end{abstract}

\section{INTRODUCTION}

I

$\mathrm{N}$ THE CONVENTIONAL fire control system (Fig. 1), present target position data obtained from the fire control director are used in the computer, to calculate target rates and accelerations. These target rates and accelerations are in turn used to predict the future target position, which is required in the solution of the fire control problem.

* Decimal classification: R116 $\times$ R537. Original manuscript received by the Institute, March 17, 1950.

† Sperry Gyroscope Company, Great Neck, L. I., N. Y.
In present-day practice an automatic-tracking radar is used to control the director, and this tracking method yields data which include random deviations from the true target position. These perturbations are called "jitter." Jitter is a matter of consequence because the computer, in computing rates and accelerations from the radar data, greatly amplifies these random deviations with the result that dispersions in the gun (or missile) orders are many times the values of the original perturbations.

With the development of modern high-altitude, highspeed, highly maneuverable targets, the computer's task has been made more difficult on two accounts: (a) The computer smoothing time has been shortened because the targets are faster and more maneuverable, and (b) the time required for projectile flight has been lengthened because longer range missiles are used. Since the amplification of the tracking perturbations is pro- 